\title{
Second-language experience modulates first- and second-language word frequency effects: Evidence from eye movement measures of natural paragraph reading
}

\author{
Veronica Whitford • Debra Titone
}

Published online: 1 November 2011

(C) Psychonomic Society, Inc. 2011

\begin{abstract}
We used eye movement measures of firstlanguage (L1) and second-language (L2) paragraph reading to investigate whether the degree of current L2 exposure modulates the relative size of L1 and L2 frequency effects (FEs). The results showed that bilinguals displayed larger L2 than L1 FEs during both early- and late-stage eye movement measures, which are taken to reflect initial lexical access and postlexical access, respectively. Moreover, the magnitude of L2 FEs was inversely related to current L2 exposure, such that lower levels of L2 exposure led to larger L2 FEs. In contrast, during early-stage reading measures, bilinguals with higher levels of current L2 exposure showed larger L1 FEs than did bilinguals with lower levels of L2 exposure, suggesting that increased L2 experience modifies the earliest stages of L1 lexical access. Taken together, the findings are consistent with implicit learning accounts (e.g., Monsell, 1991), the weaker links hypothesis (Gollan, Montoya, Cera, Sandoval, Journal of Memory and Language, 58:787-814, 2008), and current bilingual visual word recognition models (e.g., the bilingual interactive activation model plus $[\mathrm{BIA}+]$; Dijkstra \& van Heuven, Bilingualism: Language and Cognition, 5:175197, 2002). Thus, amount of current L2 exposure is a key determinant of FEs and, thus, lexical activation, in both the L1 and L2.
\end{abstract}

Electronic supplementary material The online version of this article (doi:10.3758/s13423-011-0179-5) contains supplementary material, which is available to authorized users.

V. Whitford $\cdot$ D. Titone $(\triangle)$

Department of Psychology, Centre for Research on Language,

Mind, \& Brain, McGill University,

1205 Dr. Penfield Ave.,

Montreal, QC H3A 1B1, Canada

e-mail: dtitone@psych.mcgill.ca
Keywords Eye movements and reading · Visual word recognition · Language comprehension · Psycholinguistics

Word frequency is one of the most robust variables that influence word processing. Across many measures (e.g., lexical decision, eye movement recordings) high-frequency (HF) words (home) are recognized more quickly than lowfrequency (LF) words (sock), a finding known as the word frequency effect (FE; see, e.g., Inhoff \& Rayner, 1986; Rayner \& Duffy, 1986). The effects of word frequency play a key role in determining how the mental lexicon is structured and are believed to arise as lexical forms are accessed (Rayner, 1998).

Although cognitive models differ on the exact locus of FEs, in monolinguals, FEs have often been explained through implicit learning, where repeated exposure to $\mathrm{HF}$ words strengthens lexical representations, causing them to reach threshold sooner than LF words (e.g., Monsell, 1991; but see Murray \& Forster, 2004, for an alternative explanation on rank models). Accordingly, the relationship between word frequency and lexical access time follows an asymptotic function. Consequently, repeated exposure to LF words speeds lexical access time, whereas repeated exposure to HF words minimally influences lexical access time, since HF words are at asymptote. Furthermore, increased exposure to all words functionally reduces differences in lexical access time between HF and LF words, leading to reduced FEs.

Word frequency unquestionably shapes the mental lexicon; however, most research has emphasized monolingual language processing, leaving bilingual first-language (L1) and second-language (L2) FEs less understood. Given that bilingualism is more prevalent than monolingualism globally, this imbalance is particularly noteworthy, since L2 
knowledge may mediate lexical access and, consequently, FEs, across the L1 and L2. Thus, the present study used eye movement measures of reading to examine whether FEs differ for L1 and L2 reading as a function of individual differences in L2 exposure.

Whether cognitive models of monolingual language processing can be extended to accommodate bilingual FEs may elucidate whether a common mechanism underlies both monolingual and bilingual FEs. Regarding L1 FEs, implicit learning models would predict larger L1 FEs in highly proficient bilinguals, as compared with both monolinguals and less proficient bilinguals, since highly proficient bilinguals have relatively less L1 exposure. Less proficient bilinguals and monolinguals would have similar L1 FEs, assuming similar levels of L1 exposure. Regarding L2 FEs, implicit learning models would predict reduced differences between L1 and L2 FEs for highly proficient bilinguals, assuming comparable levels of L1 and L2 exposure. Less proficient bilinguals would have larger L2 FEs, as compared with both L1 FEs and L2 FEs in highly proficient bilinguals, since L2 words are less likely to have reached asymptotic learning, given their reduced L2 exposure.

Analogous to implicit learning models, the weaker links hypothesis (Gollan, Montoya, Cera, \& Sandoval, 2008), also known as the frequency-lag hypothesis (Gollan et al., 2011), posits that relative to monolinguals, bilinguals have weaker links between word forms and their lexical representations due to reduced exposure to each language. Accordingly, divided frequency of language use weakens lexical organization, leading to longer lexical access times and, consequently, larger FEs in the lesser used language. The weaker links hypothesis makes predictions similar to those of implicit learning models: larger bilingual versus monolingual FEs, because bilinguals are not exclusively exposed to one language; larger L2 versus L1 FEs in bilinguals, because bilinguals generally have less L2 exposure; and larger L1 FEs in highly proficient bilinguals versus less proficient bilinguals, because highly proficient bilinguals have relatively less L1 exposure.

While most evidence of weaker links comes from language production studies, several comprehension studies suggest that the weaker links hypothesis extends to bilingual reading. Lemhöfer et al. (2008) found larger L2 versus monolingual $\mathrm{FEs}$, using a progressive demasking task; however, they did not evaluate bilinguals with respect to L1 processing. Similarly, Duyck, Vanderelst, Desmet, and Hartsuiker (2008) examined L1 and L2 FEs in L1dominant bilinguals versus monolinguals, using both L1 and L2 lexical decision tasks. Bilinguals showed larger L2 versus L1 FEs; however, no L1 differences arose between bilinguals and monolinguals. This finding contradicts the weaker links hypothesis and may have arisen because the bilinguals tested were highly L1 dominant or because lexical decision may lack sensitivity for detecting such differences. Recently, Gollan et al. (2011) examined FEs in English monolinguals, L2-dominant Spanish-English bilinguals, and L1-dominant Dutch-English bilinguals with exclusively English materials, using lexical decision and eye movement measures of sentence reading. Interestingly, only Dutch-English bilinguals showed L2 FEs during lexical decision. In contrast, eye movement reading measures failed to show differential FEs across the monolingual and bilingual groups.

Thus, the extant literature using standard cognitive tasks suggests that L2 FEs in bilingual readers are larger than L1 FEs in monolingual readers and that L2 FEs are larger than L1 FEs within and across bilingual readers. However, it is unclear whether these results extend to longer texts and whether they covary with graded differences in L1 and L2 exposure among bilinguals.

To address these issues, we used eye movement measures of paragraph reading to examine both L1 and L2 FEs in L1-dominant bilinguals who had varying ratios of current L1/L2 exposure. Eye movement measures are ideal for investigating these issues because they are highly naturalistic, do not require overt, artificial decisions, and can track early and late stages of reading across measures (Rayner, 2009).

Our first goal was to determine whether L2 FEs are larger than L1 FEs and whether the relative size of L2 FEs changes as a function of L2 experience. Our second goal was to determine whether the relative size of L1 FEs also changes as a function of L2 experience, thus assessing whether the weaker links hypothesis extends to bilingual comprehension.

\section{Method}

Participants

One hundred twenty-five bilinguals (75 English-French, 50 French-English) participated. Participants were McGill undergraduates and individuals from the Montreal community, who received course credit or monetary compensation. Participants had normal or corrected-to-normal vision and no self-reported history of speech, learning, or hearing disorders.

All participants' first-acquired language was also their dominant language, as determined by a language questionnaire modeled after the LEAP-Q (Marian, Blumenfeld, \& Kaushanskaya, 2007). Using this questionnaire, we assessed self-reported current percentages of time exposed to L1 versus L2. Eight participants with current L2 exposure levels greater than $50 \%$ were excluded from 
analyses, resulting in 117 participants (73 English-French, 44 French-English).

\section{Materials}

Stimuli were target words from two paragraphs, taken from the Government of Canada Web site (www.canada.gc.ca). Paragraphs were representative natural texts that individuals may encounter in daily life and were officially translated into English and French. The first paragraph contained 139 words in English and 167 words in French, and the second paragraph contained 129 words in English and 167 words in French. The paragraphs were coded for key linguistic variables, including word length, frequency, and contextual predictability. English and French word frequencies (subtitle word frequencies per million words) were obtained from the Brysbaert and New (2009) corpus of the English Lexicon Project (Balota et al., 2007) and the LEXIQUE database (New, Pallier, Ferrand, \& Matos, 2001), respectively. Contextual predictability was assessed using a cumulative Cloze task involving a separate set of 22 native English speakers and 22 native French speakers (following Miellet, Sparrow, \& Sereno, 2007). The paragraphs were matched on all linguistic variables (all $p \mathrm{~s}>.05$ ), except that the French version of the second paragraph was slightly more predictable than the English version (.36 vs. .27 Cloze probability; see Table 1). Given that the paragraphs were translation equivalents, this small but significant difference in predictability may have arisen due to inherent differences between English and French. For example, gender markings might slightly advantage French over English, where previously gender-marked words might help predict upcoming, consistently gender-marked words.

\section{Apparatus}

We used an Eye-Link 1000 tower-mounted system, with a sampling rate of $1 \mathrm{kHz}$ (SR-Research, Ontario, Canada). Although viewing was binocular, eye movements were recorded from the right eye only. Paragraphs were presented on a 21-in. ViewSonic CRT monitor, positioned $71 \mathrm{~cm}$ from participants, using Experiment Builder software. Text was presented in yellow, 14-point Courier New font on a black background. The paragraphs were doublespaced on a single screen, with three characters subtending approximately $1^{\circ}$ of visual angle.

\section{Procedure}

Participants read one paragraph version in English and a second paragraph version in French. The order of presentation of English and French paragraphs was counter- balanced across participants, as was assignment of each paragraph to a language condition. Participants were instructed to read silently and at their normal pace for comprehension. Eye movements were calibrated using a standard 9-point grid. To ensure that participants maintained attention while reading, three simple comprehension questions per paragraph were presented. The comprehension questions were answered with $93 \%$ average accuracy.

\section{Results}

Following prior work (Miellet et al., 2007; Pollatsek, Reichle, \& Rayner, 2006), words at the beginning and end of every line of text were removed from analyses. Proper nouns, repeated words, function words, cognates, and interlingual homographs were also excluded. The two English paragraphs and the two French paragraphs were combined in the analyses, totalling 100 words in each language. Subtitle word frequency values were log transformed to normalize their distribution. Fixations less than $100 \mathrm{~ms}$ in duration were excluded, resulting in $1.8 \%$ data loss.

Both early- and late-stage eye movement measures were examined (Rayner, 2009). Early-stage measures, taken to reflect the initial processes of lexical access, included firstfixation duration, gaze duration, and skipping rate. Latestage measures, taken to reflect higher-order processes of postlexical access (semantic integration and revision), included proportion of regressions into a word and total reading time. For the sake of brevity, we discuss in detail the results for gaze duration and total reading time; however, the results for the other measures are available in the online supplement. All eye movement measures are defined in Table 2.

The data were analyzed using linear mixed effects models within the lme4 package (version 0.999375-35) of R (version 2.13.1; Baayen, Davidson, \& Bates, 2008; Bates, 2007; R Development Core Team, 2010). The same model was applied to each eye movement measure. Participants and items were random factors (random intercepts only), and paragraph language (treatment coded: L1 vs. L2; L1 = baseline), frequency (continuous), and current L2 exposure (continuous) were fixed factors. Several control predictors were also included to account for variance due to differences in word length (continuous), contextual predictability (continuous), L2 age of acquisition (AoA) (continuous), and participant native language (treatment coded: English vs. French; English = baseline). To ensure that reading behavior was not altered by differences in contextual predictability across the English and French versions of the second paragraph, contextual predictability was included in the highest-order interaction in a separate 
Table 1 Word length, frequency, and contextual predictability values for the English and French versions of each paragraph

\begin{tabular}{llllll}
\hline Linguistic Variable & English Version $M$ & French Version $M$ & $d f$ & $t$ Value & $p$ Value \\
\hline Paragraph 1 & & & & & \\
$\quad$ Word length & 4.99 & 4.83 & 304 & 0.12 & $>.05$ \\
Frequency & 5431.91 & 5252.96 & 304 & 0.19 & $>.05$ \\
Contextual predictability & 0.26 & 0.26 & 304 & .05 & $>.05$ \\
Paragraph 2 & & & & & \\
$\quad$ Word length & 5.23 & 5.48 & 294 & 1.09 & $>.05$ \\
Frequency & 4773.04 & 5635.30 & 294 & 0.90 & $>.05$ \\
Contextual predictability & 0.27 & 0.36 & 294 & 2.03 & $<.05$ \\
\hline
\end{tabular}

set of analyses. To reduce collinearity, all predictors were centered. Maximum correlations among main effects were under .32 for all eye movement measures (see Table 3). Markov chain Monte Carlo (MCMC) sampling tests $(n=$ $10,000)$ were used to obtain $p$-values for all fixed factors, except skipping rate and proportion of regressions.

Frequency and current L2 exposure were analyzed continuously in all models; however, to facilitate interpretation of the data, we dichotomized them using a median split in Table 4 only. Frequency was divided into HF words (log subtitle word frequency $>2.45, n=99$ words) and LF words ( $\log$ subtitle word frequency $<2.45, n=101$ words). Similarly, current L2 exposure was divided into high L2 exposure (more than $30 \%, n=57$ participants) and low L2 exposure ( $30 \%$ or less, $n=60$ participants).

Gaze duration

A main effect of paragraph language occurred $(b=52.48, S E=$ $4.43, p_{\mathrm{MCMC}}<.001$ ), where gaze durations were shorter during L1 versus L2 reading ( 292 vs. $345 \mathrm{~ms}$, respectively) (see Table 5). A main effect of frequency also occurred $(b=-6.60$, $\left.S E=1.80, p_{\mathrm{MCMC}}<.001\right)$, where gaze durations were shorter for $\mathrm{HF}$ versus LF words (256 vs. $330 \mathrm{~ms}$, respectively).

A three-way interaction between paragraph language, frequency, and current L2 exposure occurred $(b=0.95, S E=$ $\left.0.17, p_{\mathrm{MCMC}}<.001\right)$, indicating that FEs differed across the L1 and L2 for the same participants. These effects were not driven by the second paragraph's differences in contextual predictability, since contextual predictability did not affect the highest-order interaction $\left(b=0.13, S E=0.96, p_{\mathrm{MCMC}}=.90\right)$. A subanalysis of $\mathrm{L} 1$ reading revealed near-significant greater gaze duration differences between LF and HF words in high versus low L2 exposure bilinguals $(b=-0.16, S E=0.09$, $p_{\mathrm{MCMC}}=.06 ; 55 \mathrm{~ms}$ vs. $36 \mathrm{~ms}$, respectively). This effect was fully significant for first-fixation duration $\left(p_{\mathrm{MCMC}}<\right.$ .05 ; see the online supplement). Thus, during early-stage reading, high versus low L2 exposure bilinguals exhibited larger L1 FEs, consistent with implicit learning models and the weaker links hypothesis. This pattern was reversed during L2 reading: high L2 exposure bilinguals showed smaller gaze duration differences across LF and HF words than did low L2 exposure bilinguals $(b=0.77, S E=0.14$, $p_{\text {MCMC }}<.001 ; 72$ vs. $125 \mathrm{~ms}$, respectively). This suggests that L2 experience modulates the magnitude of FEs, such that less L2 exposure leads to larger L2 FEs. Interestingly, high L2 exposure bilinguals showed reduced differences in the magnitude of L2 versus L1 FEs ( 72 vs. $55 \mathrm{~ms}$, respectively). In contrast, low L2 exposure bilinguals showed much larger L2 versus L1 FEs (125 vs. $36 \mathrm{ms,}$ respectively). The partial effects of frequency and current L2 exposure across the L1 and L2 for gaze duration are plotted in Fig. 1.

\section{Total reading time}

A main effect of paragraph language occurred $(b=105.80$, $\left.S E=7.64, p_{\mathrm{MCMC}}<.001\right)$, where total reading times were

Table 2 Definitions of early- and late-stage eye movement measures included in the analyses

\section{Eye Movement Measure Definition}

Early stage

First-fixation duration

Gaze duration

Skipping rate

Late stage

Proportion of regressions in

Total reading time
The duration of the fixation made when the eyes land on a word for the first time, irrespective of the total number of fixations made on the word

The sum of all fixation durations on a word during the first pass The percentage of words not fixated on during the first pass

The proportion of words regressed into after initially looking away The sum of all fixation durations (including refixations) on a word 
Table 3 Maximum correlations among main effects for each eye movement measure

\begin{tabular}{ll}
\hline Eye Movement Measure & $\begin{array}{l}\text { Maximum Correlation } \\
\text { Among Main Effects }\end{array}$ \\
\hline First-fixation duration & .11 \\
Gaze duration & .11 \\
Skipping rate & .32 \\
Proportion of regressions in & .12 \\
Total reading time & .12 \\
\hline
\end{tabular}

shorter during L1 versus L2 reading (431 vs. $538 \mathrm{ms,}$ respectively). A main effect of frequency also occurred $(b=$ $\left.-13.74, S E=3.08, p_{\mathrm{MCMC}}<.001\right)$, where total reading times were shorter for HF versus LF words (350 vs. $509 \mathrm{~ms}$, respectively).

Again, a three-way interaction between paragraph language, frequency, and current L2 exposure occurred $\left(b=0.79, S E=0.30, p_{\mathrm{MCMC}}<.01\right)$, where FEs differed across the L1 and L2 for the same participants. Contextual predictability did not affect the highest-order interaction $(b=$ $\left.-0.92, S E=1.65, p_{\mathrm{MCMC}}=.57\right)$, demonstrating that reading behavior did not strategically shift given the second paragraph's differences in contextual predictability. A subanalysis of $\mathrm{L} 1$ reading revealed no differences in L1 total reading times between LF and HF words across the two bilingual groups $\left(b=-0.07, S E=0.18, p_{\mathrm{MCMC}}=.70 ; 130\right.$ vs. $125 \mathrm{~ms}$, respectively). Thus, late-stage measures fail to support the weaker links hypothesis, since differential L1 FEs were not found. However, significant differences in L2 total reading times across LF and HF words were found for high versus

Table 4 Means (in milliseconds) and standard deviations (in parentheses) for all eye movement measures during first-language (L1) and second-language (L2) reading, across current L2 exposure levels (high L2 exposure $>30 \%$; low L2 exposure $<30 \%$ ) and log low L2 exposure bilinguals $\left(b=0.69, S E=0.23, p_{\mathrm{MCMC}}<\right.$ $.01 ; 169$ vs. $207 \mathrm{~ms}$, respectively). Thus, L2 FEs are modulated by individual differences in current L2 exposure. The partial effects of frequency and current L2 exposure across the L1 and L2 for total reading time are plotted in Fig. 1.

\section{Discussion}

Both early- and late-stage eye movement measures of reading revealed larger L2 than L1 FEs, suggesting that word frequency has a more robust effect during initial L2 lexical access, which then mediates higher-order processes of postlexical access (e.g., semantic integration). Previously unexplored, these effects were found for the same group of participants as a function of variations in current L2 exposure. Moreover, the magnitude of L2 FEs was inversely related to current L2 exposure levels, such that lower L2 exposure levels led to larger L2 FEs.

Interestingly, during early-stage L1 reading, high L2 exposure bilinguals showed larger L1 FEs than did low L2 exposure bilinguals. In contrast to L2 FEs, the absence of L1 FEs during late-stage reading suggests that word frequency has a more specific effect on the initial stages of L1 lexical access. Unlike in Duyck et al. (2008), who did not find that L2 knowledge mediates L1 FEs in bilinguals, the present study shows that L1 FEs are sensitive to variations in L2 exposure. These differences may be due to the nature of the task used or the type of bilinguals studied (i.e., highly L1-dominant bilinguals), which are most comparable to the low L2 exposure bilinguals in our study.

subtitle word frequencies (low frequency $[\mathrm{LF}]<2.45$; high frequency $[\mathrm{HF}]>2.45$ ). Resulting frequency effects (FEs; i.e., differences between LF and HF words) are presented in italics

\begin{tabular}{|c|c|c|c|c|c|c|c|c|}
\hline \multirow[t]{3}{*}{ Eye Movement Measure } & \multicolumn{4}{|l|}{ L1 } & \multicolumn{4}{|l|}{ L2 } \\
\hline & \multicolumn{2}{|c|}{ High L2 Exposure $n=57$} & \multicolumn{2}{|c|}{ Low L2 Exposure $n=60$} & \multicolumn{2}{|c|}{ High L2 Exposure $n=57$} & \multicolumn{2}{|c|}{ Low L2 Exposure $n=60$} \\
\hline & HF Words & LF Words & HF Words & LF Words & HF Words & LF Words & HF Words & LF Words \\
\hline First-fixation duration & $219(76)$ & $237(102)$ & $233(100)$ & $236(94)$ & $228(89)$ & $245(97)$ & $224(87)$ & $253(105)$ \\
\hline FEs & 18 & & 3 & & 17 & & 29 & \\
\hline Gaze duration & $247(94)$ & $302(165)$ & $259(152)$ & $295(158)$ & $254(121)$ & $326(192)$ & $261(126)$ & $386(281)$ \\
\hline FEs & 55 & & 36 & & 72 & & 125 & \\
\hline Skipping rate & $0.17(0.38)$ & $0.04(0.20)$ & $0.18(0.38)$ & $0.05(0.21)$ & $0.11(0.32)$ & $0.04(0.19)$ & $0.11(0.31)$ & $0.03(0.18)$ \\
\hline FEs & -0.13 & & -0.13 & & -0.07 & & -0.08 & \\
\hline Proportion of regressions in & $0.29(0.46)$ & $0.22(0.41)$ & $0.28(0.45)$ & $0.23(0.42)$ & $0.31(0.46)$ & $0.24(0.42)$ & $0.32(0.47)$ & $0.24(0.43)$ \\
\hline FEs & -0.07 & & -0.05 & & -0.07 & & -0.08 & \\
\hline Total reading time & $313(193)$ & $443(301)$ & $330(268)$ & $455(323)$ & 345 (199) & $514(393)$ & $398(300)$ & 605 (459) \\
\hline FEs & 130 & & 125 & & 169 & & 207 & \\
\hline
\end{tabular}


Table 5 Effect sizes $(b s)$, standard errors $(S E s)$, and $p_{\mathrm{MCMC}}$ values for linear mixed effects models of gaze duration and total reading time

\begin{tabular}{|c|c|c|c|c|c|c|}
\hline & \multicolumn{3}{|c|}{ Gaze Duration } & \multicolumn{3}{|c|}{ Total Reading Time } \\
\hline & $b$ & $S E$ & $p_{\mathrm{MCMC}}$ & $b$ & $S E$ & $p_{\mathrm{MCMC}}$ \\
\hline \multicolumn{7}{|l|}{ Fixed Effects } \\
\hline Paragraph language $(\mathrm{L} 1, \mathrm{~L} 2)^{\mathrm{a}}$ & 52.48 & 4.44 & $0.0001 * * *$ & 105.80 & 7.64 & $0.0001 * * *$ \\
\hline Frequency (log subtitle) & -6.60 & 1.80 & $0.0001 * * *$ & -13.74 & 3.08 & $0.0001 * * *$ \\
\hline Current L2 exposure (\% time) & 0.37 & 0.42 & 0.3810 & 1.20 & 0.94 & 0.2344 \\
\hline Paragraph language $*$ frequency & -15.79 & 2.27 & $0.0001 * * *$ & -22.78 & 3.88 & $0.0001 * * *$ \\
\hline Paragraph language * current L2 exposure & -2.32 & 0.36 & $0.0001 * * *$ & -4.40 & 0.62 & $0.0001 * * *$ \\
\hline Frequency $*$ current L2 exposure & -0.16 & 0.13 & 0.1876 & -0.05 & 0.21 & 0.8030 \\
\hline Paragraph language $*$ frequency $*$ current L2 exposure & 0.95 & 0.18 & $0.0001 * * *$ & 0.79 & 0.30 & $0.0094 * *$ \\
\hline Paragraph language $*$ frequency $*$ current L2 exposure $*$ contextual predictability ${ }^{\mathrm{b}}$ & 0.13 & 0.96 & 0.9024 & -0.92 & 1.65 & 0.5716 \\
\hline \multicolumn{7}{|l|}{ Control predictors } \\
\hline Word length & 10.21 & 0.95 & $0.0001 * * *$ & 26.81 & 1.63 & $0.0001 * * *$ \\
\hline Contextual predictability & -20.38 & 10.14 & $0.0456^{*}$ & -82.08 & 17.34 & $0.0001 * * *$ \\
\hline L2 age of acquisition (years) & 1.38 & 1.08 & 0.1916 & -0.12 & 2.56 & 0.8190 \\
\hline Participant native language (English, French) ${ }^{\mathrm{c}, \mathrm{d}}$ & -2.17 & 10.33 & 0.8782 & -5.49 & 16.50 & 0.6950 \\
\hline (Intercept) & 291.92 & 8.64 & $0.0020 * *$ & 432.10 & 16.65 & $0.001 * * *$ \\
\hline Random Effects & \multicolumn{3}{|c|}{ Variance } & \multicolumn{3}{|c|}{ Variance } \\
\hline Subject & \multicolumn{3}{|c|}{$2,482.93$} & \multicolumn{3}{|c|}{$16,518.20$} \\
\hline Item & \multicolumn{3}{|l|}{83.18} & \multicolumn{3}{|c|}{200.87} \\
\hline Residual & \multicolumn{3}{|c|}{$35,206.13$} & \multicolumn{3}{|c|}{$104,272.19$} \\
\hline
\end{tabular}

${ }^{\text {a }}$ Contrasts were treatment coded; model assumes L1 as the baseline across conditions.

${ }^{\mathrm{b}}$ Contextual predictability does not significantly affect the highest-order interaction.

${ }^{\mathrm{c}}$ Contrasts were treatment coded; model assumes English as the baseline across conditions.

${ }^{\mathrm{d}}$ Participant native language (English vs. French) does not significantly affect the highest-order interaction.

${ }^{*} p_{\mathrm{MCMC}}<.05$

$* * p_{\mathrm{MCMC}}<.01$

$* * * p_{\mathrm{MCMC}}<.001$

The L1 FE results are consistent with the weaker links hypothesis (Gollan et al., 2008), suggesting that L1 FEs observed in bilingual language production extend to bilingual reading. On this point, our data contrast with those of Gollan et al. (2011), who did not find differences in FEs across bilingual groups. However, sentence reading may have created a less demanding cross-language load than did paragraph reading, which may have affected the manifestation of FEs. Furthermore, Gollan et al. compared only L2 FEs in bilinguals with L1 FEs in monolinguals, rather than evaluating bilingual effects on L1 and L2 reading for the same set of participants given individual differences in relative L1 versus L2 exposure.

The present study is also consistent with current models of bilingual language processing (e.g., the bilingual interactive activation model plus (BIA+; Dijkstra \& van Heuven, 2002). In BIA+, word frequency modulates resting activation levels of lexical representations and, thus, initial lexical access (see also Dahan,
Magnuson, \& Tanenhaus, 2001, for FEs during spokenword recognition). Furthermore, BIA+'s temporal delay assumption posits that L2 words have subjectively lower frequencies than do L1 words, leading to longer lexical access times and, consequently, larger L2 FEs. Accordingly, for less proficient bilinguals, BIA+ would predict larger L2 FEs than L1 FEs, consistent with our findings of larger L2 FEs than L1 FEs in low L2 exposure bilinguals. For highly proficient bilinguals, BIA + would again predict larger L2 than L1 FEs; however, there would be reduced differences between L1 and L2 FEs, since activation thresholds across languages are comparable when subjective word frequencies are comparable. The finding that high L2 exposure bilinguals displayed reduced differences in L1 versus L2 Fes, relative to low L2 exposure bilinguals, supports this prediction. These findings are also consistent with implicit learning models (e.g., Monsell, 1991), but do not support existing rank models (Murray \& Forster, 2004). 
L1

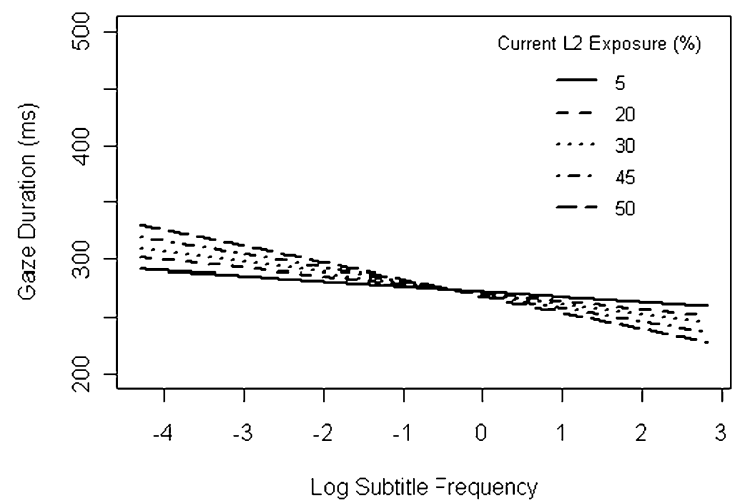

L1

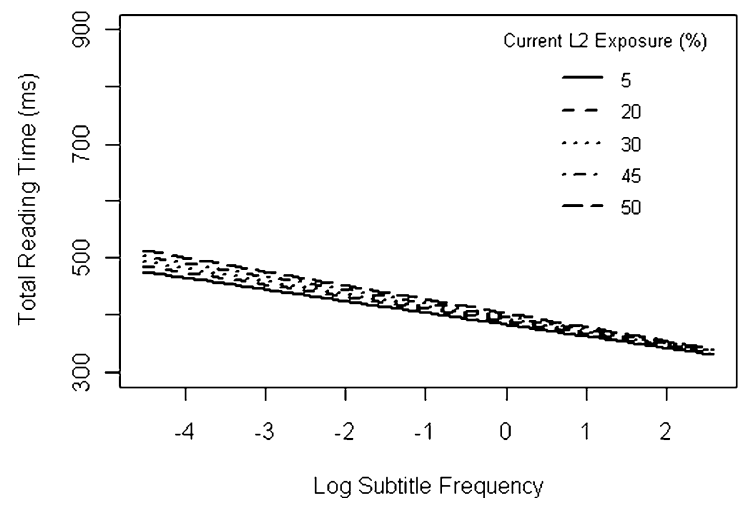

Fig. 1 Graphical representation of the partial effects of frequency and current second-language (L2) exposure across the first language (L1) and L2 for gaze duration (top right and top left panels, respectively). Graphical representation of the partial effects of frequency and current L2 exposure across the L1 and L2 for total reading time (bottom right and bottom left panels, respectively). Quantile regression lines of model-fitted values are presented, for which the 5\% L2 exposure line

If weaker L1 lexical-semantic links are an important property of the bilingual lexicon, they may also contribute to other cross-language effects previously reported. For example, bilinguals demonstrate cross-language effects during cognate and interlingual homograph processing (e.g., Libben \& Titone, 2009; Schwartz \& Kroll, 2006; Van Assche, Duyck, Hartsuiker, \& Diependaele, 2009), where lexical information from both languages is dually activated (Dijkstra \& van Heuven, 2002; Green, 1998). Weaker L1 lexicalsemantic links may function synergistically with duallanguage activation, making highly proficient bilinguals more susceptible to cross-language activation during L1 processing (e.g., Elston-Güttler, Paulmann, \& Kotz, 2005). Accordingly, highly proficient bilinguals should encounter more cross-language competition in the L1 than should less proficient bilinguals, who would exclusively encounter L2 cross-language competition (consistent with Libben \& Titone, 2009; Titone, Libben, Mercier, Whitford, \& Pivneva, 2011). Consequently, increased cross-language competition in highly proficient bilinguals
L2

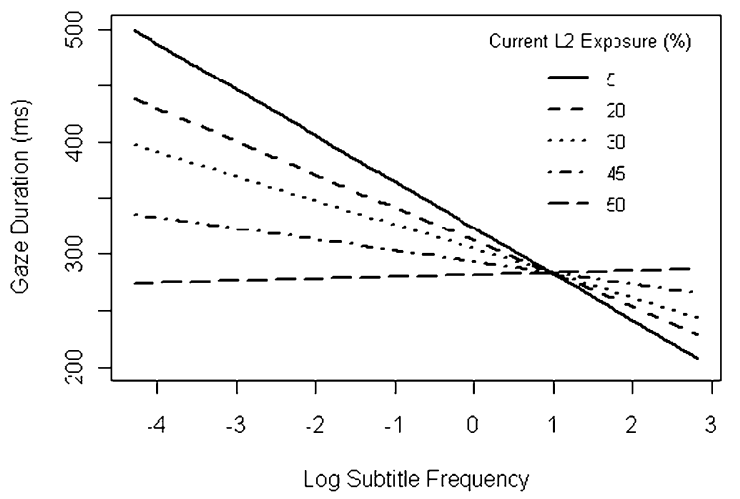

L2

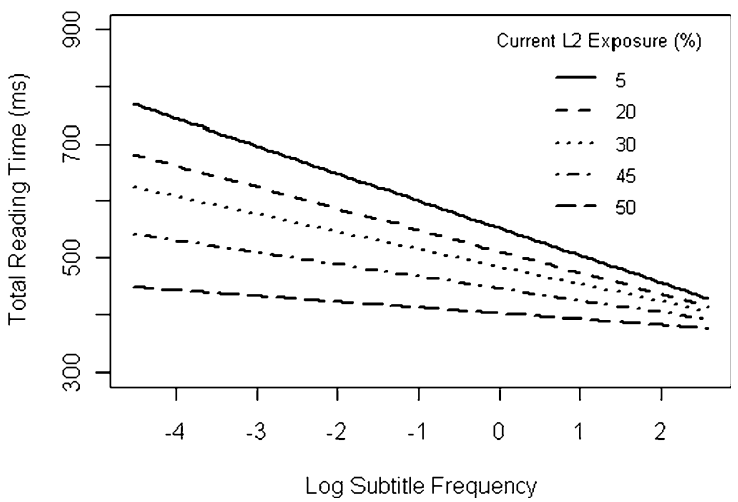

represents participants at the 0 percentile (based on the overall distribution of data); the $20 \% \mathrm{~L} 2$ exposure line represents participants at the 25th percentile; the $30 \%$ L2 exposure line represents participants at the 50th percentile; the $45 \%$ L2 exposure line represents participants at the 75 th percentile; and the 50\% L2 exposure line represents participants at the 100th percentile

may heighten demands on executive function during language processing. Thus, if Bialystok and colleagues are correct, highly proficient bilinguals should reap more executive control benefits than should less proficient bilinguals (Bialystok \& Craik, 2010). Whether weaker L1 lexical-semantic links drive dual activation constitutes an important topic of future work.

To conclude, the relative amount of bilinguals' L1 and L2 experience is a key determinant of lexical activation and, consequently, FEs during bilingual reading. Thus, L2 knowledge and usage affect how bilinguals process L2 words and, more provocatively, how they process L1 words (see also Titone et al., 2011; Van Assche et al., 2009).

Author Note This research was supported by the following grants to D. Titone: Natural Sciences and Engineering Research Council of Canada (NSERC) Discovery Award, the Canada Research Chairs (CRC) Program, the Canadian Foundation for Innovation (CFI), and the Centre for Research on Language, Mind and Brain (CRLMB). We also gratefully acknowledge additional support from an NSERC Undergraduate Student Research Award awarded to V. Whitford and 
a Fonds Québecois de recherche sur la nature et les technologies (FQRNT) masters fellowship awarded to V. Whitford. Correspondence concerning this article should be addressed to D. Titone, Department of Psychology, McGill University, 1205 Doctor Penfield Ave., Montreal, H3A 1B1, Canada (e-mail: dtitone@psych.mcgill.ca).

\section{References}

Baayen, R. H., Davidson, D. J., \& Bates, D. M. (2008). Mixed effects modeling with crossed random effects for subjects and items. Journal of Memory and Language, 59, 390-412.

Balota, D. A., Yap, M. J., Cortese, M. J., Hutchison, K. A., Kessler, B., Loftis, B.,...Treiman, R. (2007). The English Lexicon Project. Behavior Research Methods, 39, 445-459.

Bates, D. M. (2007). Linear mixed model implementation in lme4. Unpublished manuscript. University of Wisconsin, Madison.

Bialystok, E., \& Craik, F. I. M. (2010). Cognitive and linguistic processing in the bilingual mind. Current Directions in Psychological Science, 19, 19-23.

Brysbaert, M., \& New, B. (2009). Moving beyond Kučera and Francis: A critical evaluation of current word frequency norms and the introduction of a new and improved word frequency measure for American English. Behavior Research Methods, 41, 977-990.

Dahan, D., Magnuson, J. S., \& Tanenhaus, M. K. (2001). Time course of frequency effects in spoken-word recognition: Evidence from eye movements. Cognitive Psychology, 42, 317-367.

Dijkstra, T., \& van Heuven, W. J. B. (2002). The architecture of the bilingual word recognition system: From identification to decision. Bilingualism: Language and Cognition, 5, 175-197.

Duyck, W., Vanderelst, D., Desmet, T., \& Hartsuiker, R. J. (2008). The frequency effect in second-language visual word recognition. Psychonomic Bulletin \& Review, 15, 850-855.

Elston-Güttler, K. E., Paulmann, S., \& Kotz, S. A. (2005). Who's in control? Proficiency and L1 influence on L2 processing. Journal of Cognitive Neuroscience, 17, 1593-1610.

Gollan, T. H., Montoya, R. I., Cera, C., \& Sandoval, T. C. (2008). More use almost always means a smaller frequency effect: Aging, bilingualism, and the weaker links hypothesis. Journal of Memory and Language, 58, 787-814.

Gollan, T. H., Slattery, T. J., Goldenberg, D., van Assche, E., Duyck, W., \& Rayner, K. (2011). Frequency drives lexical access in reading but not in speaking: The frequency-lag hypothesis. Journal of Experimental Psychology: General, 140, 186-209.

Green, D. W. (1998). Mental control of the bilingual lexico-semantic system. Bilingualism: Language and Cognition, 1, 67-81.

Inhoff, A. W., \& Rayner, K. (1986). Parafoveal word processing during eye fixations in reading: Effects of word frequency. Perception \& Psychophysics, 40, 431-439.
Lemhöfer, K., Dijkstra, T., Scriefers, H., Baayen, R. H., Grainger, J., \& Zwitserlood, P. (2008). Native language influences on word recognition in second language: A megastudy. Journal of Experimental Psychology: Learning, Memory, and Cognition, $34,12-31$

Libben, M. R., \& Titone, D. A. (2009). Bilingual lexical access in context: Evidence from eye movements during reading. Journal of Experimental Psychology: Learning, Memory, and Cognition, 35, 381-390.

Marian, V., Blumenfeld, K. H., \& Kaushanskaya, M. (2007). The Language Proficiency and Experience Questionnaire (LEAP-Q): Assessing language profiles in bilinguals and multilinguals. Journal of Speech, Language, and Hearing, 50, 940-967.

Miellet, S., Sparrow, L., \& Sereno, S. C. (2007). Word frequency and predictability effects in reading French: An evaluation of the E-Z Reader model. Psychonomic Bulletin \& Review, 14, 762-769.

Monsell, S. (1991). The nature and locus of word frequency effects in reading. In D. Besner \& G. W. Humphreys (Eds.), Basic processes in reading: Visual word recognition (pp. 148-197). Hillsdale, NJ: Erlbaum.

Murray, W. S., \& Forster, K. I. (2004). Serial mechanisms in lexical access: The rank hypothesis. Psychological Review, 111, 721-756.

New, B., Pallier, C., Ferrand, L., \& Matos, R. (2001). Use base de donnés lexicales du français contemporain sur internet: LEXIQUE 3. L'Année Psychologique, 101, 447-462.

Pollatsek, A., Reichle, E. D., \& Rayner, K. (2006). Tests of the E-Z Reader model: Exploring the interface between cognition and eye movement control. Cognitive Psychology, 52, 1-56.

Rayner, K. (1998). Eye movements in reading and information processing: 20 years of research. Psychological Bulletin, 124, $372-422$.

Rayner, K. (2009). Eye movements and attention in reading, scene perception, and visual search. Quarterly Journal of Experimental Psychology, 62, 1457-1506.

Rayner, K., \& Duffy, S. A. (1986). Lexical complexity and fixation times in reading: Effects of word frequency, verb complexity, and lexical ambiguity. Memory \& Cognition, 14, 191-291.

R Development Core Team. (2010). $R$ : A language and environment for statistical computing. (Version 2.13.1). Vienna: R Foundation for Statistical Computing. Retrieved from http://www.R-project.org

Schwartz, A. I., \& Kroll, J. F. (2006). Bilingual lexical activation in sentence context. Journal of Memory and Language, 55, $197-$ 212.

Titone, D., Libben, M., Mercier, J., Whitford, V., \& Pivneva, I. (2011). Bilingual lexical access during L1 sentence reading: The effects of L2 knowledge, semantic constraint, and L1-L2 intermixing. Journal of Experimental Psychology: Learning, Memory, and Cognition.

Van Assche, E., Duyck, W., Hartsuiker, R. J., \& Diependaele, K. (2009). Does bilingualism change native-language reading? Psychological Science, 20, 923-927. 